\title{
Effets et risques liés à l'utilisation des champs magnétiques dans les explorations par résonance magnétique nucléaire chez l'homme*
}

\author{
P. GALLE ${ }^{\star \star}$ et J.F. CAVELLIER ${ }^{\star \star}$ \\ (Manuscrit reçu le 20 décembre 1984)
}

\begin{abstract}
RÉSUME
Un profond bouleversement est apparu ces dernières années dans les méthodes physiques d'exploration du corps humain. Parmi ces méthodes, celle utilisant le phénomène de résonance magnétique nucléaire suscite un intérêt tout particulier pour plusieurs raisons. Tout d'abord, il est possible en utilisant le phénomène de résonance magnétique nucléaire du proton, d'obtenir in vivo des images des organes internes avec une remarquable précision, et de mettre ainsi en évidence des lésions qu'il ètait impossible de visualiser autrefois par les méthodes classiques utilisant les rayons $X$ ou les ultrasons. Outre cet aspect purement morphologique, la résonance magnétique nucléaire permet aussi d'obtenir, toujours in vivo, des renseignements sur la nature chimique des structures internes et sur le métabolisme de certains éléments. Enfin, dans ces explorations, le malade n'est soumis à aucune radiation ionisante. II est soumis, par contre, à un champ magnétique constant de valeur élevée, à des champs magnétiques variables, et à des radiations électromagnétiques de haute fréquence. Les risques encourus par le malade dans ces conditions semblent minimes et aisément contrôlables. II est néanmoins apparu opportun d'en faire le bilan et de préciser quels sont les effets susceptibles d'apparaître dans un organisme vivant soumis à ces champs et à ces radiations non ionisantes.
\end{abstract}

\section{ABSTRACT}

The physical methods of dynamic clinical investigation have been largely modified lately. Medical imaging by nuclear magnetic resonance (NMR) is of particular interest for several reasons. 1) Very precise in vivo images of internal organs are obtained that demonstrate lesions impossible to detect so far with the classical methods using X-rays or ultrasounds. 2) Besides this purely morphologic aspect, NMR supplies in vivo data on the chemical nature of internal structures and on the metabolism of some elements. 3) Finally, the patient is not exposed to any ionising radiation. $\mathrm{He}$ is, however, exposed to a constant high magnetic field, to variable magnetic fields and to high-

* Conférence présentée lors de l'Assemblée générale de la Société française de radioprotection le 22 juin 1984.

* Laboratoire de biophysique. Faculté de médecine, 8, rue du Général Sarrail, 94010 Créteil. 
frequency electromagnetic radiation. The risks for the patients are very low and easily controlled. Nevertheless, it seems now advisable to assess the situation and to review the effects likely to occur in a living being exposed to magnetic fields and non-ionising radiation.

\section{INTRODUCTION}

Après les premières constatations d'Arsene d'Arsonval en 1896 [2], les effets biologiques des champs magnétiques ont donné lieu à une littérature très abondante et controversée.

Depuis une vingtaine d'années, une nouvelle impulsion a été donnée à ces études avec le développement, dans l'industrie ou la recherche, d'instruments susceptibles de soumettre le personnel à des champs magnétiques élevés ou à des radiations électromagnétiques de haute fréquence (accélérateurs de particules, réacteurs de fusion, chambres à bulles, radars, fours industriels).

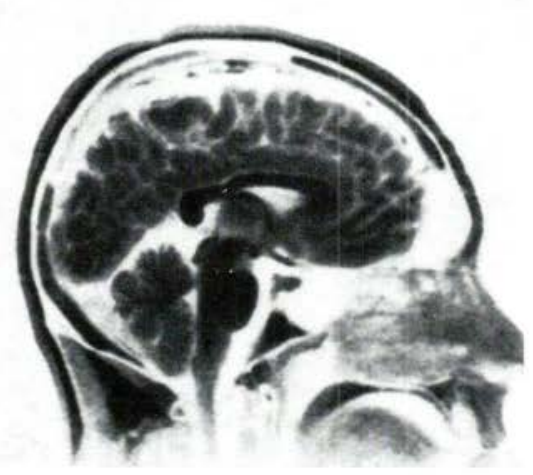

Fig. 1. - Image obtenue par résonance magnétique nucléaire sous un champ magnétique de 5000 gauss. II s'agit d'une coupe sagittale médiane d'un crâne. Le cerveau et ses circonvolutions ainsi que le cervelet sont bien mis en évidence, de même que le bulbe rachidien, l'origine de la moelle et les cavités ventriculaires (scanner CGR).

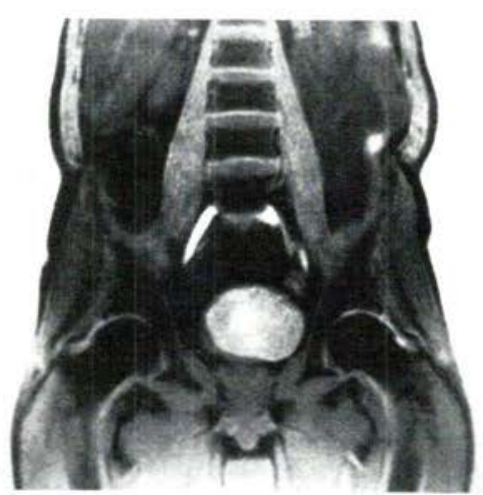

Fig. 2. - Image obtenue au niveau de la partie inférieure de l'abdomen. Chaque vertèbre est clairement individualisèe ainsi que chaque disque intervertébral, la partie inférieure du foie, les faisceaux musculaires, les deux têtes femorales et la vessie (scanner CGR, 5000 gauss). 
Très récemment enfin, de nouvelles méthodes d'exploration du corps humain se sont développées en médecine; elles utilisent le phénomène de résonance magnétique nucléaire et permettent entre autres d'obtenir in vivo des images des organes internes avec une remarquable précision. La qualité des images actuelles (fig. 1 et 2), la richesse des informations qu'elles contiennent dépassent déjà largement les possibilités des meilleurs scanner $X$, et un développement important de ces méthodes dans le domaine du diagnostic médical est attendu ces prochaines années.

Cet important développement justifie qu'une attention particulière soit maintenant portée au problème des risques potentiels encourus par le malade lors de ces explorations et que des seuils de sécurité soient définis. Dans ces explorations, le malade n'est soumis à aucun rayonnement ionisant, mais il est, par contre, soumis à la fois à : 1) un champ magnétique constant et uniforme de vaieur élevée $(0,04$ à 2 tesla) ; 2) des champs magnétiques variables dans l'espace (gradient de champ) et avec le temps, et 3 ) des rayonnements de haute fréquence (2 à $14 \mathrm{MHz}$ ).

On se limitera dans cet exposé à l'étude des risques encourus dans ces conditions.

\section{LE CHAMP MAGNETIQUE CONSTANT ET UNIFORME}

Dans une exploration in vivo, les champs utilisés varient actuellement suivant les instruments de 400 à 10000 gauss pour l'imagerie du proton et des champs plus intenses, jusqu'à 20000 gauss $(2 \mathrm{~T})$ sont utilisés pour l'analyse spectrométrique localisée. Ces valeurs sont donc 1000 à 40000 fois plus élevées que celles du champ magnétique terrestre $(0,5$ gauss). Dans ces conditions, des effets très divers peuvent apparaître dans la matière vivante.

L'influence d'un champ magnétique sur la matière peut se manifester de deux façons: 1) l'apparition de forces mécaniques qui s'appliquent sur toute particule chargée en mouvement (forces de Lorentz) et qui tendent à courber leur trajectoire, et 2) une interaction du champ avec les structures magnétiques.

Dans la matière vivante, ces deux effets peuvent se manifester puisqu'il existe, d'une part, un grand nombre de particules chargées en mouvement (ions atomiques et moléculaires) et, d'autre part, des microstructures cellulaires et des molécules qui selon les cas peuvent être dia, para ou ferro-magnétiques.

\section{La force de Lorentz et la conduction de l'influx nerveux}

Rappelons qu'une particule chargée q animée d'une vitesse $\vec{v}$ placée dans un champ $B$ est soumise à une force $F$ perpendiculaire à la fois au champ et à la vitesse et telle que :

$$
\vec{F}=q \vec{v} \wedge \vec{B}
$$

dont le sens est donné par la figure 3 : 


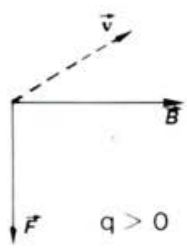

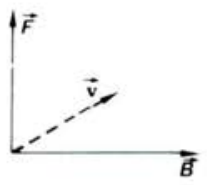

$q<0$
Fig. 3. - Forces subies par des particules de charges positive et négative, animées de la même vitesse $\vec{v}$ dans un champ magnétique $\vec{B}$ perpendiculaire a $\vec{v}$.

Cette force, qui a comme effet de courber la trajectoire de toute particule chargée en mouvement, pourra se manifester dans les milieux biologiques aqueux, par exemple sur les ions solubles participant au mouvement brownien ou sur le flux d'ions transmembranaire induit lors du passage de l'influx nerveux. Reno [30] et Kolta [19] ont ainsi décrit d'importantes modifications de la vitesse de conduction nerveuse sous l'influence de champs magnétiques fixes de $1 \mathrm{~T}$. Ces résultats n'ont cependant pas été confirmés par d'autres expérimentateurs travaillant dans des conditions contrôlées sur des préparations isolées de nerfs de crustacés ou de sciatique de grenouille [12, 33].

L'effet attendu des forces de Lorentz à ce niveau peut, d'ailleurs, n'être que négligeable, compte tenu du très faible parcours moyen des ions en agitation thermique dans le milieu aqueux (quelques angströms au maximum) et de la faible distance du parcours du flux d'ions transmembranaire (environ 70 angströms) (cf. annexe 1).

On considère, actuellement, que le seuil nécessaire pour obtenir une réduction mesurable $(10 \%)$ de la vitesse de conduction de l'influx nerveux est très élevé : $24 \mathrm{~T}[21,38]$, soit des valeurs nettement plus élevées que celles utilisées dans une exploration par RMN.

\section{L'effet Hall et les anomalies de l'électrocardiogramme}

Des anomalies de l'onde T de l'électrocardiogramme (ECG) apparaissent chez le mammifère (rats, chiens, singes) lors de l'exposition à un champ magnétique statique $[6,13,36]$. Cet effet a été largement confirmé ; il disparaît dès la fin de l'exposition. Le seuil d'apparition se situe à 0,3 T et les anomalies augmentent avec l'intensité du champ.

Des anomalies de cette nature, apparaissant pour des valeurs du champ identiques à celles utilisées pour l'imagerie du corps entier, sont a priori préoccupantes et nécessitent une attention particulière. L'explication la plus plausible de ce phénomène est liée à l'effet Hall susceptible d'apparaître au niveau des gros vaisseaux (cf. annexe 2).

Le calcul développé dans l'annexe 2 montre que, pour un champ de $1 \mathrm{~T}$, un débit sanguin de $5 \mathrm{l} / \mathrm{min}$, une artère de $3 \mathrm{~cm}$ de diamètre, la différence de potentiel $\triangle \mathrm{V}=\mathrm{V}_{\mathrm{A}}-\mathrm{V}_{\mathrm{B}}$ de part et d'autre de l'artère est $\triangle \mathrm{V}=4,71 \mathrm{mV}$, soit un champ électrique d'environ $0,16 \mathrm{~V} / \mathrm{m}$.

Les valeurs de la différence de potentiel induite par effet Hall sont suffisantes pour être détectées par les électrodes cutanées utilisées 
pour l'enregistrement de l'ECG. Dans le cas d'une exploration par RMN, le champ magnétique est dirigé suivant l'axe du corps et une différence de potentiel peut être induite dans tout segment de vaisseau perpendiculaire à cet axe, soit pour l'aorte au niveau de la partie médiane de la crosse. Le flux sanguin rapide, émis par la contraction ventriculaire, traverse ce segment aortique une fraction de seconde après la systole ; la différence de potentiel qui en résulte peut donc être synchrone à l'onde T et expliquer les anomalies observées à ce niveau.

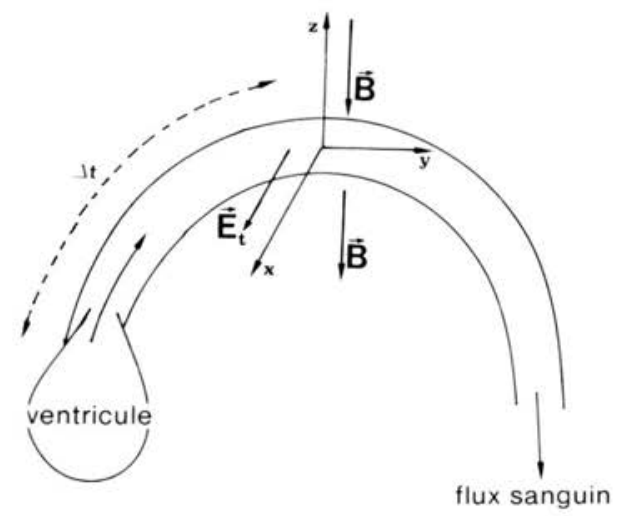

Fig. 4. - Apparition d'un champ électrique transversal $\vec{E}$, dans la crosse aortique par effet Hall sous l'effet d'un champ magnétique parallèle à l'axe du corps.

Cette interprétation des anomalies de l'électrocardiogramme semble actuellement admise par la plupart des expérimentateurs [13, 31]. Les risques attendus d'un tel phénomène sont, par contre, très faibles. On peut les apprécier en comparant les valeurs des champs électriques régnant naturellement au sein de la matière vivante avec celles créées par effet Hall. L'ordre de grandeur de la différence de potentiel existant de part et d'autre d'une membrane cellulaire est en moyenne de $70 \mathrm{mV}$ sur une épaisseur de $70 \AA$, soit un champ électrique local de $10^{7} \mathrm{~V} / \mathrm{m}$. Comparée à cette valeur du champ électrique local naturel, celle apparaissant dans l'effet Hall $(0,16 \mathrm{~V} / \mathrm{m})$ apparaît tout à fait négligeable.

Une confirmation définitive de cette interprétation des anomalies de l'ECG apparaît donc comme très souhaitable; elle permettrait d'éliminer toute probabilité de risque associé. Remarquons dans l'attente que, pour l'instant, aucun trouble du rythme cardiaque n'a été observé pour des champs de 2 T et que les anomalies de l'ECG disparaissent immédiatement après cessation de l'exposition au champ. II convient, néanmoins, de rester prudent car une exposition prolongée $(1 \mathrm{~h})$ à des champs plus intenses $(7 \mathrm{~T})$ a pu provoquer une arythmie et une diminution du rythme cardiaque [23].

\section{Effet d'un champ magnétique fixe sur les structures dia, para et ferromagnétiques}

Dans la matière vivante, toutes les molécules organiques sont diamagnétiques, certaines molécules $\left(\mathrm{O}_{2}\right)$ sont paramagnétiques et cer- 
taines microstructures peuvent être ferromagnétiques. Placées dans un champ magnétique, ces molécules ou ces microstructures seront donc soumises à une force et leur énergie sera modifiée.

1) Pour une structure ferromagnétique qui peut être considérée comme un dipôle de moment permanent $\vec{\mu}$, l'énergie d'interaction $W$ est donnée par la formule :

$$
\mathrm{W}=\vec{\mu} \overrightarrow{\mathrm{B}}
$$

Le champ magnétique a tendance à orienter la structure dans la direction du champ.

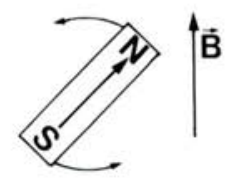

Fig. 5. - Orientation d'un dipôle magnétique dans un champ $\vec{B}$.

De telles structures ferromagnétiques (ferrites) ont èté observées en microscopie électronique dans un très grand nombre de cellules vivantes (chez des bactéries, pigeons, poissons). Sensibles au champ magnétique terrestre, elles jouent un rôle essentiel dans les phénomènes de migration, mais jusqu'à présent, aucune structure de ce type n'a été mise en évidence chez le mammifère.

2) Pour les structures dia ou paramagnétiques ne possédant pas de moment permanent, l'énergie d'interaction est donnée par la formule :

$$
W=\frac{-X V}{2 \mu_{0}} \vec{B}^{2}
$$

où $X$ est la susceptibilité magnétique, $V$ le volume et $\mu_{0}$ la perméabilité magnétique.

L'effet du champ magnétique est, ici, d'induire une polarisation magnétique dans la structure considérée. Les corps diamagnétiques $(X<0)$ se déplacent dans les régions où $B$ est le plus faible et les corps paramagnétiques $(X>0)$ se déplacent vers les régions où $B$ est plus élevé. Pour des champs de l'ordre de $1 \mathrm{~T}$, l'énergie d'interaction est beaucoup trop faible [23] pour qu'une quelconque action biologique puisse être attendue.

Certaines molécules linéaires $(A D N)$, certaines microstructures organisées (bâtonnets rétiniens, cristaux liquides) ou certaines cellules (hématies falciformes) ont des susceptibilités magnétiques anisotropes (variant avec la direction). L'effet de polarisation peut alors être suffisant pour influer sur l'orientation des molécules ou structures concernées. On a ainsi mis en évidence un effet d'orientation sur les bâtonnets rétiniens $[15,17]$ pour des champs de $1 \mathrm{~T}$ et des effets d'alignement des hématies 
falciformes [26] pour des champs de 0,35 T. Ces phénomènes sont donc observés pour des valeurs du champ du même ordre de grandeur que ceux utilisés en exploration humaine. Ces observations posent le problème d'une possible action au niveau des molécules d'intérêt biologique majeur comme l'ADN.

\section{Etudes concernant les risques génétiques et mutagènes}

Un très grand nombre de travaux ont été consacrés à ce sujet avec des résultats très contradictoires. Certains travaux ont semblé démontrer une action des champs magnétiques sur le taux de croissance cellulaire, l'induction de mutations génétiques ou la stérilité [7, 18]. II s'agit, généralement, de travaux anciens qui n'ont pas été reproduits par la suite [11]. Des travaux plus récents [35] effectués sur des bactéries n'ont pu mettre en évidence aucun effet significatif affectant le taux de survie ou le nombre de mutations. Des expériences sur les drosophiles exposées de façon continue à un champ de $0,4 \mathrm{~T}$ pendant plusieurs générations n'ont induit aucun effet détectable [25]. Aucune anomalie n'a pu être observée sur des cultures de lymphocytes humains exposés in vitro. Aucune modification génétique n'a été notée par les expérimentateurs étudiant le taux de mutations génétiques de plantes eukaryotes soumises à une exposition prolongée à des champs de 3,7 T [30] et aucune mutation n'a été observée sur des cellules de mammifères exposées à des champs identiques à ceux utilisés en exploration humaine [34].

Au total, il n'existe actuellement aucun argument permettant de supposer que des champs magnétiques intenses (de l'ordre de $1 \mathrm{~T}$ ) puissent être à l'origine d'un effet mutagène. La poursuite des travaux dans ce domaine demeure, néanmoins, souhaitable.

\section{Effets métaboliques divers}

L'influence des champs magnétiques sur le métabolisme cellulaire a été peu étudiée. Des programmes de recherche devraient sans doute être engagés dans ce domaine. II est possible, par exemple, que les phénomènes de transfert d'électrons au niveau de la chaîne des cytochromes puissent être influencés par des champs magnétiques intenses susceptibles d'entraîner des effets d'orientation moléculaire. On ne dispose d'aucune donnée à ce sujet.

De même, les phénomènes de supraconductivité évoqués dans certaines réactions enzymatiques peuvent être affectés par un champ magnétique. Néanmoins, les seuils prévus à ce niveau sont élevés et les expériences effectuées dans ce domaine, étudiant la cinétique d'un certain nombre de réactions enzymatiques soumises à des champs variant de 5 à $20 \mathrm{~T}$, n'ont pu mettre en évidence, jusqu'à présent, aucun effet significatif [29].

\section{LES VARIATIONS RAPIDES DU CHAMP MAGNETIQUE}

En exploration d'imagerie par RMN, le malade est exposé à des champs magnétiques variables en fonction du temps, champs qui viennent se 
surimposer au champ magnétique constant $\vec{B}_{0}$. Ces variations rapides du champ $\overrightarrow{\mathrm{dB}} / \mathrm{dt}$ sont à l'origine de courants induits dont l'effet au niveau des tissus est très différent de ceux précédemment étudiés. Ces champs variables ont eux-mêmes trois origines : 1) les variations du gradient de champ utilisé pour obtenir des informations spatiales ; 2) les variations accompagnant l'établissement et la coupure du champ magnétique $B_{0}$, et 3 ) les très rapides variations sinusoïdales de la composante magnétique des rayonnements de haute fréquence utilisés pour mettre en résonance les noyaux.

\section{Rappel sur l'origine des courants induits}

La loi de Faraday nous indique que toute variation du flux d'induction magnétique $\varnothing$ à travers une surface limitée par un circuit conducteur produit, dans ce circuit, , une force électromotrice d'induction u dont la valeur est donnée par:

avec $\varnothing=\iint \vec{B}_{\mathrm{S}} \overrightarrow{\mathrm{n}}_{\mathrm{o}} \mathrm{d} \mathrm{S}$

$$
u=-\frac{d \varnothing}{d t}
$$

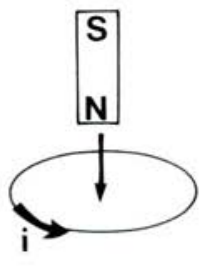

Fig. 6. - Sens du courant induit dans un circuit conducteur par une variation du champ magnétique à l'intérieur de ce circuit.

II en résulte un courant $i$ dont le sens est donné par la figure 6 et un champ électrique local $E$ tel que

$$
E=\frac{u}{2 \pi r} \text {, si r est le rayon de la boucle de courant }
$$

La densité de courant $J$ est donnée par

$$
\vec{J}=\vec{E} \sigma \quad(\sigma=\text { conductivité }) \text {, }
$$

soit une puissance instantanée $\mathrm{P}=\frac{\mathrm{dW}}{\mathrm{dt}}$ délivrée par unité de volume

$$
P=E^{2} \sigma
$$

et une énergie absorbée pendant le temps $\mathrm{t}$

$$
W=\int_{0}^{t} E^{2} \sigma d t
$$

S'agissant de l'étude des effets biologiques, les grandeurs physiques intéressantes à considérer seront différentes suivant les caractéristiques des variations de champ.

Pour des variations de haute fréquence $(10 \mathrm{MHz})$ la direction des courants induits s'inverse dix millions de fois par seconde, les effets sur 
la dépolarisation des membranes ont tendance à s'annuler, l'énergie est essentiellement dissipée sous forme thermique et la grandeur intéressante à considérer ici est la puissance absorbée. Cet effet sera étudié dans le prochain chapitre.

Si la densité de courant locale est maintenue pendant un temps suffisant dans la même direction, des effets très différents peuvent être, par contre, attendus affectant notamment la polarisation des membranes biologiques. Les grandeurs physiques intéressantes à considérer ici sont essentiellement les champs électriques locaux et les densités de courant.

Avant d'aborder ces phénomènes, précisons que le corps humain est un mauvais conducteur et surtout un conducteur très hétérogène dans lequel on trouve des volumes liquidiens macroscopiques, microscopiques et submicroscopiques faiblement conducteurs, séparés par des membranes de haute résistivité. Dans un tel conducteur, l'effet du phénomène d'induction est double : 1) excitation de courants induits à l'intérieur de régions conductrices, et 2 ) polarisation des parois isolantes. La topologie des lignes de champs et de courant sera essentiellement dépendante du gradient de la conductivité, et elles ne peuvent être définies. II est donc impossible de déterminer les valeurs exactes des champs électriques locaux et des courants induits. Seules, des valeurs très approchées peuvent être prédites. De plus, le modèle théorique très simplifié présenté ci-dessus ne tient pas compte d'effets qui peuvent être très importants ; en particulier, les courants induits réduisent le champ magnétique à l'intérieur du corps, et le flux calculé en appliquant naïvement la loi de Faraday à un conducteur circulaire virtuel à l'intérieur duquel le flux est calculé à partir du champ extérieur appliqué, conduit à surévaluer les densités de courant induit.

Un calcul de ce type [8] considérant un milieu de conductivité homogène de 0,2 siemens $/ \mathrm{m}$, une boucle de courant de $20 \mathrm{~cm}$ de diamètre, montre que la valeur de la densité de courant induite pour une variation de champ de $1 \mathrm{~T} / \mathrm{s}$ est de l'ordre de $1 \mathrm{\mu A} / \mathrm{cm}^{2}$. Cette valeur peut être comparée à celles susceptibles d'induire certains effets biologiques.

\section{- Ordre de grandeur de la densité de courant susceptible d'induire certains effets biologiques}

1) Induction d'une fibrillation cardiaque : 100 à $1000 \mu \mathrm{A} / \mathrm{cm}^{2}$;

2) Induction d'un électrochoc : $3 \mathrm{~mA} / \mathrm{cm}^{2}$ (pendant $300 \mathrm{~ms}$ ) ;

3) Induction de flash lumineux rétinien par application d'électrodes externes sur la tête: $17 \mu \mathrm{A} / \mathrm{cm}^{2}[4,8]$;

4) Densité de courant apparaissant lors du potentiel d'action d'un nerf : $3000 \mu \mathrm{A} / \mathrm{cm}^{2}$.

Les valeurs des seuils provoquant ces quatre effets ont été déterminés par application directe de courant. Les mêmes effets peuvent être attendus de l'exposition à un champ magnétique variable.

\section{- Effets biologiques observés sous l'influence de variations de champ dB/dt}

\section{$1^{\circ}$ Induction de magnétophosphènes}

Les flash lumineux rétiniens peuvent également être provoqués par des courants induits sous l'influence d'un champ magnétique variable. 
Ces "magnétophosphènes" ont été décrits par d'Arsonval en 1896 [2]. Des études récentes ont permis d'apprécier les seuils d'apparition de ces magnétophosphènes en fonction de la variation du champ magnétique $\mathrm{dB} / \mathrm{dt}$ $[3,22]$. Le seuil est de l'ordre de 2 à $5 \mathrm{~T} / \mathrm{s}$. Pour de telles variations, le calcul prévoit des densités de courants locaux de l'ordre de 2 à $5 \mu \mathrm{A} / \mathrm{cm}^{2}$, soit des valeurs très proches de celles mesurées directement lors d'application d'électrodes sur la peau.

\section{$2^{\circ}$ Modification des plaquettes sanguines}

Des anomalies des plaquettes sanguines humaines exposées in vitro à des variations de champ de $24 \mathrm{~T} / \mathrm{s}$ (sous forme d'impulsions d' $1 \mu \mathrm{s}$ avec un temps de croissance de $34 \mathrm{~ms}$ ) ont été observées au microscope électronique : dégranulation, rupture de membrane et apparition de pseudopodes [8].

\section{- Appréciation pratique des risques liés aux variations de champ}

La comparaison des valeurs de ces seuils mesurés expérimentalement avec celles des courants induits prévisibles lors d'une exploration par RMN permet d'apprécier les risques encourus possibles.

- Les densités de courant nécessaires pour induire un électrochoc sont 1000 fois supérieures à celles généralement prévues lors d'une exploration et les temps d'application nécessaires sont plus élevés. On peut raisonnablement en conclure que le risque ici est pratiquement inexistant.

- Le seuil d'induction de magnétophosphènes (2 à $5 \mathrm{~T} / \mathrm{s}$ ) est du même ordre de grandeur que les valeurs susceptibles d'être utilisées en exploration d'imagerie humaine. Aucune manifestation de ce type ne semble, cependant, avoir été observée jusqu'à présent. De toute façon, l'apparition de ces phénomènes visuels ne constitue pas en soi un danger pour le patient.

- L'induction d'une fibrillation cardiaque est un danger beaucoup plus sérieux. Une fibrillation ventriculaire peut être induite chez l'homme avec des densités de courant de l'ordre de $300 \mu \mathrm{A} / \mathrm{cm}^{2}$ [37] nécessitant probablement des variations de champ dB/dt environ 100 fois supérieures à celles induites par la plupart des instruments actuels. Malgré cette marge de sécurité apparemment suffisante, une certaine prudence s'impose car de nombreuses inconnues subsistent. En effet, le seuil de sécurité ne dépend pas seulement de la densité de courant mais également de la surface sur laquelle ce courant est appliqué et de nombreux autres facteurs tels que la forme de l'onde magnétique, sa durée et sa répétition. Ces facteurs varient considérablement suivant les différentes méthodes utilisées. Dans l'attente d'informations plus précises, il est prudent de disposer sur place de matériel de réanimation.

- L'observation d'anomalies ultrastructurales et d'activation des plaquettes sanguines pour des variations de champ de $24 \mathrm{~T} / \mathrm{s}$ pose, enfin, le problème d'une possible induction de thromboses locales. Aucun phénomène de ce type ne semble néanmoins avoir été observé jusqu'à présent.

En conclusion, on manque encore de données quantitatives suffisantes sur les différents facteurs susceptibles d'influencer les effets 
biologiques liés à une variation de champ magnétique pour que l'on puisse préciser définitivement les seuils de sécurité. Le seuil préconisé (mars 1981) par le National radiological protection board au Royaume-Uni est de $20 \mathrm{~T} / \mathrm{s}$ pour des impulsions de $10 \mathrm{~ms}$ ou plus longues; des variations supérieures à $20 \mathrm{~T} / \mathrm{s}$ semblent néanmoins autorisées pour des impulsions de durée plus courte.

Le Department of health and human service des Etats-Unis a fixé ce seuil (en 1982) à $3 \mathrm{~T} / \mathrm{s}$.

\section{LES RAYONNEMENTS DE HAUTE FREQUENCE}

Dans une exploration par RMN, le sujet est exposé à un flux de rayonnements électromagnétiques de haute fréquence, nécessaire pour l'obtention du phénomène de résonance nucléaire. La fréquence de résonance $v_{0}$ est donnée par la formule :

$$
v_{\circ}=\frac{\gamma}{2 \pi} \mathrm{B}_{0}
$$

où $\gamma$ est une caractéristique du noyau considéré (rapport gyromagnétique) et $B_{0}$ la valeur du champ magnétique constant appliqué.

Pour l'étude de la résonance du proton, les valeurs de $B_{0}$ varient en moyenne, suivant les instruments, de 0,04 à $0,35 \mathrm{~T}$ correspondant à des fréquences $v_{0}$ variant de 1,6 à $14 \mathrm{MHz}$, soit des longueurs d'onde variant de 20 à $150 \mathrm{~m}$.

\section{Effets thermiques}

Les risques attendus de l'exposition à de tels rayonnements non ionisants sont essentiellement liés à des phènomènes d'échauffement. Ces risques étaient appréciés autrefois en fonction de la valeur du flux énergétique incident. En fait, les phénomènes observés (échauffement) sont entièrement liés à l'énergie absorbée qui, à flux incident égal, peut être très différente suivant la fréquence du rayonnement incident. En exploration par RMN, il est essentiel d'utiliser des rayonnements dont l'absorption par les tissus soit aussi faible que possible permettant à la fois de recueillir les signaux émis par les parties profondes du corps humain et d'échauffer aussi peu que possible les organes.

\section{- Variation de l'énergie absorbée}

1) Avec la dimension de l'objet

L'énergie absorbée varie à la fois avec la fréquence de l'onde incidente et avec les dimensions de l'objet irradié. On a ainsi montré [14, 20] que si la composante électrique de l'onde est parrallèle à l'axe de l'objet, un phénomène de résonance apparaît lorsque la longueur de l'objet irradié est égale à $0,4 \lambda(\lambda=$ longueur d'onde). A ce niveau, on constate un maximum d'absorption. Ces variations importantes de l'énergie absorbée avec les 
dimensions de l'objet irradié expliquent qu'il n'est pas possible d'apprécier un effet biologique produit chez l'homme à partir d'expériences faites sur de petits animaux : à flux incident égal et pour une même fréquence du rayonnement, la différence des effets attendus chez l'homme et chez le rat peut être de 2 ordres de grandeur.

2) Avec la fréquence de l'onde incidente

Pour un corps de $1,75 \mathrm{~m}$, de $0,30 \mathrm{~m}$ de diamètre, la puissance spécifique absorbée (PSA) en fonction de la fréquence de l'onde incidente a l'allure suivante (fig.7, d'après [10]) pour un flux incident de $0,1 \mathrm{~mW} / \mathrm{cm}^{2}$.

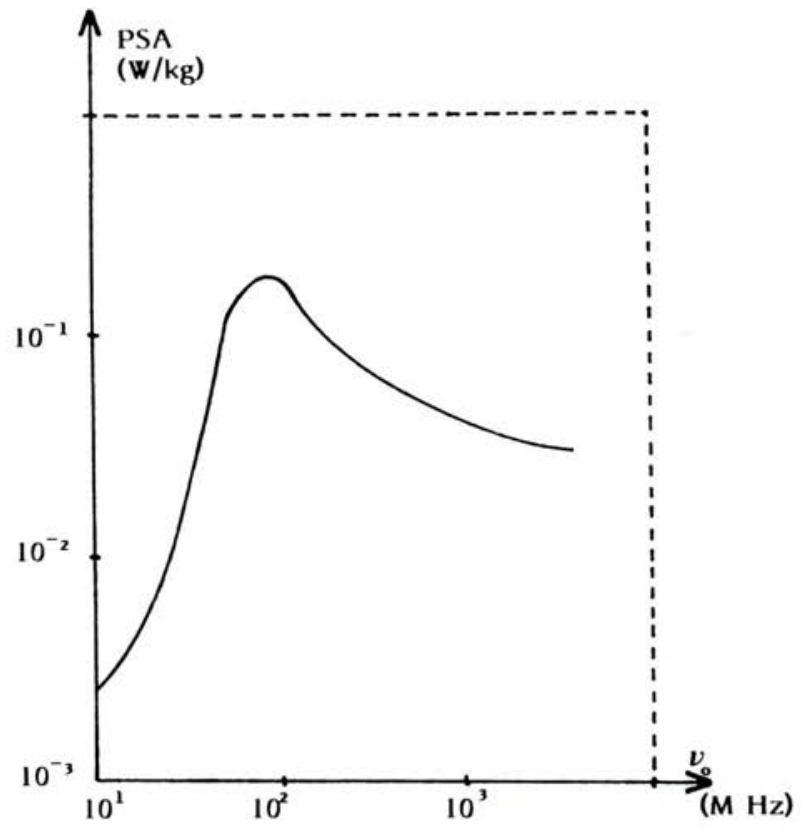

Fig. 7. - Variations de la puissance spécifique absorbée en fonction de la fréquence de l'onde incidente, pour un flux de $0,1 \mathrm{~mW} / \mathrm{cm}^{2}$.

On observe un maximum d'absorption pour une fréquence de l'ordre de $70 \mathrm{MHz}(0,4 \lambda=2 \mathrm{~m})$. Pour un flux incident de $1 \mathrm{~mW} / \mathrm{cm}^{2}$, la puissance absorbée est de $0,1 \mathrm{~W} / \mathrm{kg}$ à $50 \mathrm{MHz}$. Pour les fréquences habituellement utilisées en RMN, la puissance absorbée est environ 50 fois plus faible. La très rapide élévation de l'absorption de 10 à $50 \mathrm{MHz}$ est l'un des principaux facteurs limitant l'utilisation de champs magnétiques $B_{0}$ élevés pour l'imagerie in vivo du proton. 


\section{- Les conditions de sécurité en exploration humaine}

La puissance dissipée par le métabolisme chez l'homme varie de $1,5 \mathrm{~W} / \mathrm{kg}$ au repos à $15 \mathrm{~W} / \mathrm{kg}$ lors d'un excercice intense. Ces valeurs donnent un ordre de grandeur des seuils à ne pas dépasser lorsqu'une puissance supplémentaire est délivrée par une irradiation de champ de haute fréquence. On peut calculer [8] l'élévation de température $\Delta \Theta$ des tissus absorbant une quantité donnée d'énergie par la formule :

$$
\triangle \Theta=\frac{P S A \times t}{4186 \times h}
$$

où h est la chaleur spécifique du tissu.

Pour une PSA égale à $4 \mathrm{~W} / \mathrm{kg}$, t à $10 \mathrm{~min}, \mathrm{~h}$ à $0,83 \mathrm{kcal} / \mathrm{kg} /{ }^{\circ} \mathrm{C}$, on obtient une élévation de température $\triangle \Theta=0,7^{\circ} \mathrm{C}$.

Chez l'être vivant irradié dans un volume donné, cette élévation de température sera atténuée par dissipation thermique par conduction hors du volume irradié et vers l'extérieur. $\Delta \Theta$ dépendra donc du volume irradié et des conditions extérieures de température et d'humidité.

Le problème se pose également de savoir si cette élévation thermique est homogène ou non dans le milieu irradié et de prédire si oui ou non des points chauds peuvent être observés. Pour les fréquences utilisées dans l'exploration in vivo, les longueurs d'onde sont trop élevées par rapport aux dimensions des organes ou cavités présentes dans le corps pour que des phénomènes de résonance puissent apparaître. On peut néanmoins prévoir que pour des organes peu irrigués (cristallin, testicules) l'élévation de la température locale sera supérieure à celle des tissus voisins. De même, l'échauffement attendu est plus élevé en superficie qu'en profondeur.

Des expériences faites sur l'animal suggèrent que le seuil d'induction de la cataracte se situe aux alentours de $43^{\circ} \mathrm{C}$, soit une élévation de $6{ }^{\circ} \mathrm{C}$ seulement.

Citons, enfin, à ce niveau que la présence de prothèses métalliques au sein des tissus, absorbant fortement l'énergie incidente, peut être à l'origine d'une élévation importante de la température locale.

\section{Normes de sécurité}

Les normes de l'American national standard institute (ANSI) [16] fixent le seuil de puissance spécifique absorbée à $0,4 \mathrm{~W} / \mathrm{kg}$ sur l'ensemble du corps. L'élévation thermique prévue dans ces conditions est inférieure à $1{ }^{\circ} \mathrm{C}$. Pour des temps d'exposition inférieurs à $10 \mathrm{~min}$, Budinger [8] propose un seuil plus élevé : $4 \mathrm{~W} / \mathrm{kg}$ pour des fréquences inférieures à $10 \mathrm{MHz}$.

\section{$2^{\circ}$ Effets non thermiques}

Un certain nombre d'effets non thermiques des rayonnements de haute fréquence ont été décrits chez l'animal et sur diverses préparations biologiques, par exemple, l'alignement de particules colloïdales ou de cellules sanguines pour des rayonnements à des fréquences de 1 à $100 \mathrm{MHz}$. II semble s'agir d'attraction électrostatique entre les particules rendues 
dipolaires par la composante électrique du champ. Un tel effet semble peu probable in vivo car les intensités nécessaires pour l'induction de ce phénomène sont nettement plus élevées que celles utilisées en exploration humaine [32]. De même, ont été décrites des modifications du transport de calcium ou encore des modifications de perméabilité de la barrière cérébro-méningée $[1,27]$. Ces expériences n'ont pas été confirmées par la suite [24, 28]. Néanmoins, ces observations incitent à une certaine prudence et il est souhaitable que d'autres travaux soient effectués dans ce domaine.

\section{L'EXPERIENCE ACTUELLE CHEZ L'HOMME}

Au Royaume-Uni, 40 volontaires sains ont été soumis à des expositions à des champs magnétiques dans des conditions identiques à celles des explorations cliniques et pendant des temps prolongés (90 min). Les expériences ont été parfois répétées plusieurs fois chez le même sujet. Aucune manifestation objective ou subjective n'a été décelée. Les explorations hématologiques, biochimiques, électrocardiographiques et encéphalographiques pratiquées avant et après exposition ont toutes été négatives.

Par ailleurs, plusieurs milliers de malades ont subi dans le monde des explorations d'imagerie du proton ou de spectrométrie localisée. Un accident a été observé aux Etats-Unis chez un malade porteur d'une prothèse métallique intracérébrale. Mise à part cette exception qui aurait dû aisément être évitée, jusqu'à présent les seules manifestations généralement ubservées sont une certaine claustrophobie, parfois une sensation de chaleur ou une sensation de gêne au niveau de prothèses dentaires.

On peut donc raisonnablement penser qu'une exploration par résonance magnétique nucléaire est une méthode non invasive comportant des risques immédiats extrêmement faibles. Quant à la probabilité de risques à long terme, si elle n'est pas entièrement éliminée, elle est extrêmement peu probable.

S'agissant néanmoins d'une exploration nouvelle, il est indispensable de rester encore vigilant et un certain nombre de règles doivent être respectées. Jusqu'à plus ample information, il est recommandé d'éviter toute exploration chez des malades porteurs de stimulateurs cardiaques, chez ceux ayant des antécédents d'épilepsie et chez la femme enceinte. La plus grande prudence s'impose également chez les cardiaques et les malades porteurs de prothèse métallique. On doit, enfin, pouvoir disposer sur place de matériels de réanimation.

\section{NORMES RECOMMANDEES}

Le tableau suivant résume les normes recommandées par deux organismes officiels aux Etats-Unis et au Royaume-Uni. 
TABLEAU I

Seuils recommandés en RMN in vivo par le National radiological protection board (NRPB) du Royaume-Uni (1981) et par le Department of health and human service (DHHS), USA (1982)

\begin{tabular}{|l|l|l|}
\hline \multirow{2}{*}{$\begin{array}{l}\text { Champ magnétique } \\
\text { statique }\end{array}$} & \multicolumn{1}{|c|}{ NRPB } & \multicolumn{1}{c|}{ DHHS } \\
\cline { 2 - 3 } $\begin{array}{l}\text { Champ magnétique } \\
\text { variable }\end{array}$ & $\begin{array}{l}<20 \text { tesla/s pour des } \\
\text { impulsions }>10 \mathrm{~ms} ; \text { le seuil } \\
\text { peut être plus élevé pour des } \\
\text { impulsions plus courtes }\end{array}$ & $\begin{array}{l}<3 \text { tesla/s (exposition totale } \\
\text { partielle du corps) } \\
\text { ou partielle du corps) }\end{array}$ \\
\hline $\begin{array}{l}\text { Rayonnement de } \\
\text { haute fréquence } \\
\text { (15 MHz) }\end{array}$ & $\begin{array}{l}\text { Exposition telle que la puis- } \\
\text { sance moyenne absorbée } \\
\text { dans l'ensemble du corps soit } \\
<70 \text { W } \\
\text { Des réserves sont faites sur la possible apparition de points } \\
\text { chauds dans certaines structures critiques (cristallin) }\end{array}$ & $\begin{array}{l}\text { Exposition telle que la puis- } \\
\text { sance moyenne absorbée soit } \\
<\text { W/kg }\end{array}$ \\
\hline
\end{tabular}

On remarquera que ces normes sont différentes de celles recommandées pour les travailleurs susceptibles d'être exposés quotidiennement à des champs magnétiques statiques intenses et qui ont été définies (règles non officielles) dans différents laboratoires et notamment au Stanford linear accelerator en Californie (tableau II) et par Vyalov en URSS (tableau III). Ces normes peuvent être appliquées pour le personnel travaillant quotidiennement dans un service médical d'exploration par résonnance magnétique nucléaire.

TABLEAU ॥

Stanford linear accelerator

\begin{tabular}{|l|l|l|l|}
\hline \multicolumn{1}{|c|}{ Champ } & Temps dexposition & \multicolumn{1}{|c|}{ Région du corps } & Commentaires \\
\hline $0.02 \mathrm{~T}$ & non définie & $\begin{array}{l}\text { corps entier } \\
\text { corps entier } \\
0.2 \mathrm{~T}\end{array}$ & quelques minutes \\
$0.2 \mathrm{~T}$ & non définie & mains et tête & $\begin{array}{l}\text { périodes prolongées pos- } \\
\text { sibles }\end{array}$ \\
$2 \mathrm{~T}$ & quelques minutes & mains et tête & \\
\hline
\end{tabular}

TABLEAU ॥

Recommandations préconisées en URSS (Vyalov)

(Champs magnétiques statiques uniformes et gradients de champ)

\begin{tabular}{|c|c|c|}
\hline Champ & Région du corps & Commentaires \\
\hline $\begin{array}{l}0,03 \mathrm{~T} \\
0,07 \mathrm{~T} \\
0,0005 \mathrm{~T} / \mathrm{cm} \\
\text { à } 0,002 \mathrm{~T} / \mathrm{cm} \\
0,001 \mathrm{~T} / \mathrm{cm} \\
\text { à } 0.002 \mathrm{~T} / \mathrm{cm}\end{array}$ & $\begin{array}{l}\text { corps entier } \\
\text { mains } \\
\text { corps entier } \\
\text { mains }\end{array}$ & $\begin{array}{l}\text { exposition continue en méde- } \\
\text { cine du travail } \\
\text { gradients de champ } \\
\text { en médecine du travail }\end{array}$ \\
\hline
\end{tabular}




\section{CONCLUSION}

L'expérience actuelle, portant sur un très grand nombre d'explorations par résonance magnétique nucléaire effectuées sans qu'aucun incident notable n'ait pu être observé sauf une exception qui aurait pu aisément être évitée, incite à une très bonne confiance dans l'innocuité de cet examen.

Par principe, néanmoins, une certaine vigilance demeure nécessaire en ce qui concerne à la fois les risques immédiats (cardiaques, circulatoires) et tardifs (risque mutagène, cataracte).

Les normes préconisées doivent être considérées comme un guide pratique applicable actuellement dans le seul domaine de l'exploration médicale et ne doivent pas être appliquées dans d'autres contextes tels que l'exposition du public ou l'exposition journalière en médecine du travail. Ces normes seront susceptibles d'être reconsidérées lorsque nos connaissances sur l'effet biologique des champs magnétiques seront mieux établies. L'établissement de ces seuils de sécurité ne signifie pas qu'il soit actuellement ou qu'il sera interdit de les dépasser. Dans ce domaine, comme dans toute exploration médicale, l'indication de l'examen doit être pris individuellement pour chaque malade, la responsabilité du médecin étant d'évaluer le risque encouru pour ce malade particulier par rapport au bénéfice escompté pour le patient.

\section{REFERENCES}

[1] ALBERT E.N. Proceedings of the Symposium on the Biological effects and measurements of radiofrequency/microwaves, 1977, 294-304.

[2] ARSONVAL M.A. d' C.R. Sci. Biol., 1896, 3. 451.

[3] BARLOW H.B., KOHN H.I., WALSH E.G. Am. J. Physiol., 1947, 148. 372-375.

[4] BARLOW H.B., KOHN H.I., WALSH E.G. J. Physiol., 1947, 148. 376-381.

[5] BAUM J.W., SCHAIRER L.A., LINDAHL. K.L. In : Magnetic field effects on biological systems (Tenforde T.S., Ed.). New York: Plenum press, 1979, vol. 1. 22-24.

[6] BEISCHER D.E. Aerospace Med., 1964, 35. 939-944.

[7] BREWER H.B. Biophys J., 1979, 28, 305.

[8] BUDINGER T.F. In : Nuclear Magnetic resonance imaging in medicine (Kaufman, Crooks and Margulis, Eds.). Tokyo : Igaku-Shoin, 1981, vol. 1, 207-231.

[9] BUDINGER T.F. Lawrence Radiation laboratory report UCL - 18347, 1968.

[10] DURNEY C.H., JOHNSON C.C., BARBER P.W., MASSOUDI H., ISKANDER M.F. LORDS J.L., RYSER D.K., ALLEN J., MITCHELL J.C. In : Radiofrequency radiation dosimetry handbook (2nd Ed). Brooks Air Force Base, Texas 78235.

[11] EISELEN B.S., BOUTELL H.M., BIGGS M.W. Aerospace Med.. 1961, 32, 383.

[12] GAFFEY C.T., TENFORDE T.S. Bioelectromagnetics, 1980, 1. 208.

[13] GAFFEY C.T., TENFORDE T.S., DEAN E.E. Bioelectromagnetics, 1980, 1, 209.

[14] GANDHI O.P., HAGMANN M.J. In : Abstracts URSI Int. Symposium on the Biological effects of electromagnetic waves. Airlie, Va., Oct. 1977, 51.

[15] GEACINTOV N.E., VAN NOSTRAND F., BECKER J.F., TINKEL J.B. Biochim. Biophys. Acta, 1972, 267, 65-79.

[16] GOULD J.L. La recherche, 1983, 14, 186-197. 
[17] HONG F.T., MAUZerall D., MAURO A. Proc. Nat. Acad. Sci. USA, 1971, 68, $1283 \cdot 1285$.

[18] KIMBOL G.C. J. Bacteriol. 1937, 35, 109.

[19] KOLTA P. Acta Physiol. Acad. Sci. Hung. 1973, 43. 89-94.

[20] KUCIA H.R. IEEE Trans. Instrum. Measurement IM 1972, 21, 412.

[21] LIBOFF R.L. Biophys. J., 1965, 5, 845-853.

[22] LOVSUND P., NILSSON S.E.G., REUTER T.. OBERG P. Med. Biol. Eng. Comput., $1980,18,326-334$.

[23] MANSFIELD P., MORRIS P.G. In : NMR imaging in biomedicine. New York : Academic press, 1982, 297-331.

[24] MERRITT J.H., CHAMNESS A.F., ALLEN S.J. Radiat. Environ. Biophys., 1978 , $15,367-377$.

[25] MULAY I.L., MULAY L.N. In : Biological effects of magnetic fields (TENFORDE T.S. Ed.). New York: Plenum press, 1964, Vol. 1.

[26] MURAYAMA M. Nature, 1965, 206. 420-422.

[27] OSCAR K.I., HAWKINS T.D. Brain Res., 1977, 126, 281-293.

[28] PRESTON E., VAVASOUR E.J., ASSENHEIM H.M. Brain Res., 1979, 174. 109-117.

[29] RABINOVITCH B., MALING J.E., WEISSBLUTH M. Biophys. J., 1967, 7. 187-204.

[30] RENO V.R. NASA Report NAMI-1089, 1969.

[31] SALLES-CUNHA S.X., BATTOCLETTI J.H., SANCES A. IEEE Proc., 1980, 68, 149-155.

[32] SCHWAN H.P. Therapeutic heat and cold, 2. ed. (Licht S.H., Ed.). New-Haven : E. Licht, 1965, 63-125.

[33] SCHWARTZ J.L. IEEE Trans. Biomed. Eng. BME, 1978 25, 467-473.

[34] SCHWARTZ J.L., CROOKS L.E. Am. J. Roentgenol., 1982, 139, 583-585.

[35] THOMAS A., MORRIS P.G. Br. J. Radiol., 1981, 54, 615.

[36] TOGAWA T., OKAI O., OSHIMA M. Med. Biol. Eng., 1967, 5. 169-170.

[37] WATSON A.B., WRIGHT J.S., LOUGHMAN J. Med. J. Aust., 1973, 1. 1179-1180.

[38] WIKSWO J.P., BARACH J.P. IEEE Trans. Biomed. Eng., BME 1980, 27, 722-723.

\section{ANNEXE 1}

\section{Evaluation de l'effet d'un champ magnétique uniforme sur la diffusion passive des ions}

La vitesse moyenne dagitation thermique des ions calcium dans les milieux biologiques (à $37^{\circ} \mathrm{C}$ ) est donnèe par :

$$
\frac{1}{2} M \widetilde{\sim}^{2}=\frac{3}{2} R T
$$

soit $\bar{v}=440 \mathrm{~m} / \mathrm{s}$.

Dans les champs magnétiques maximaux de $2 \mathrm{~T}$ envisagés ici, la force de Lorentz apparaissant lors d'une translation perpendiculaire à $\mathrm{B}$ d'un ion $\mathrm{Ca}^{++}$sera de :

$$
\begin{gathered}
|\vec{F}|=2 \text { e } \bar{v} \text { B } \\
|\vec{F}| \simeq 2,816.10^{-16} \text { newton }
\end{gathered}
$$


II en résulte qu'un élément de trajectoire d'un ion calcium, normalement rectiligne, est transformé en un arc de cercle de rayon moyen :

$\bar{R}=2 \mathrm{~m} \bar{v}$ e $\mathrm{B}$ rayon cyclotron, minimal dans le cas où la vitesse est perpendiculaire à $\vec{B}$. Ici : $R=4,56 \cdot 10^{-5} \mathrm{~m}=45,10^{3} \mathrm{~nm}$

Le rayon de courbure est très grand comparé, par exemple, à l'épaisseur de la membrane cellulaire (env. $10 \mathrm{~nm}$ )

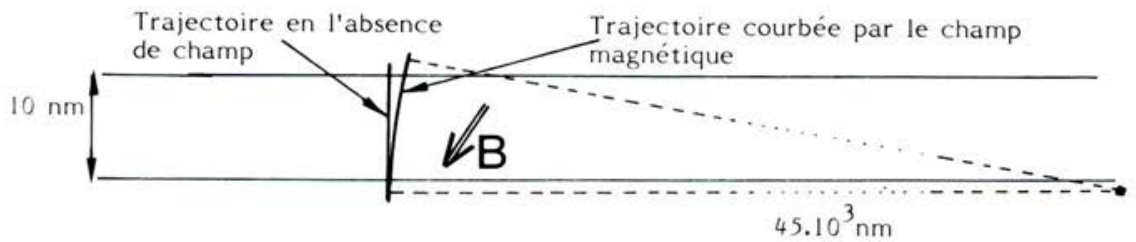

Le déplacement latéral d'un ion calcium au cours de la traversée d'une membrane cellulaire serait au maximum de $1,1.10^{-3} \mathrm{~nm}$, c'est-à-dire moins d'un millième du diamètre attribué à l'ion calcium.

\section{ANNEXE 2}

\section{Rappel sur l'effet Hall}

Dans un conducteur, les porteurs de charges en mouvement, par exemple les ions positifs et négatifs véhiculés dans le flux aortique, sont soumis à la force de Lorentz dès qu'un champ magnétique ayant une composante perpendiculaire à la direction du flux est appliqué.

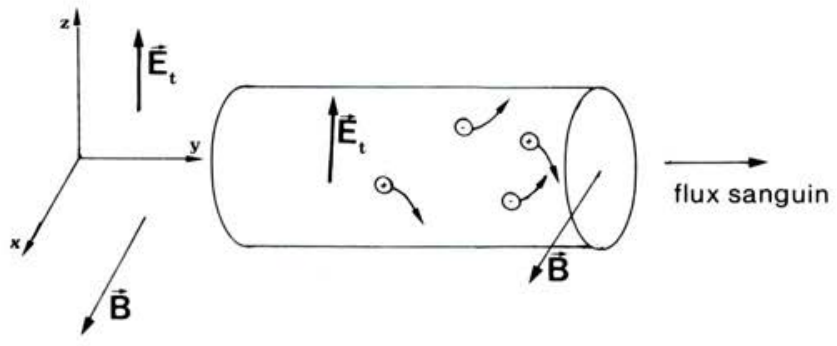

Fig. 8. - Apparition d'un champ électrique transversal dans un flux d'ions soumis à un champ magnétique (effet Hall).

II en résulte que la distribution des charges est modifiée. Sur la figure 8 les charges + s'accumulent vers le bas, les charges - vers le haut, et un champ électrique transverse est créé. Si le champ magnétique $\vec{B}$ est dirigé perpendiculairement et en avant de la feuille, le champ électrique créé $\vec{E}_{1}$ est dirigé vers le haut. Sa valeur est déterminée par l'égalité

$$
q \vec{E}_{t}=-q \vec{v} \Lambda \vec{B}
$$


indiquant qu'à l'équilibre la force électrostatique $q E_{t}$ annule en tous points la force de Lorentz. La valeur du champ $E_{t}$ est donc :

$$
\vec{E}_{\mathrm{t}}=-\vec{v} \wedge \vec{B}
$$

La différence de potentiel à travers un vaisseau de diamètre $d$ est donnée, dans la direction perpendiculaire au champ magnétique, par :

$$
\begin{gathered}
\Delta V=\int_{0}^{d}-\vec{E} d \vec{i} \\
\Delta V=\int_{0}^{d}(\vec{v} \wedge \vec{B}) d \vec{i}
\end{gathered}
$$

$\Delta V=\vec{B} \cdot \int_{0}^{d}-\vec{v} \wedge d \vec{\imath}$ l'intégrale étant calculée le long du diamètre du vaisseau. En valeur absolue, la différence de potentiel est égale au produit du champ magnétique par la surface de la parabole des vitesses dans le vaisseau ; en désignant par $\bar{v}$ la vitesse moyenne d'écoulement définie par $\bar{v}=1 / d \int_{0}^{d} v$. dl, il vient :

$$
\Delta \mathrm{V}=\mathrm{B} \overline{\mathrm{v}} \mathrm{d}[\mathrm{8}]
$$

Si l'on suppose un écoulement laminaire dans le vaisseau, la distribution de la vitesse en fonction de la distance $r$ à l'axe du vaisseau suit une loi du type

$$
v(r)=\frac{4 v_{0}}{d^{2}}\left(\frac{d^{2}}{4}-r^{2}\right)
$$

conforme à la loi de Poiseuille, où $v_{0}$ est la vitesse maximale, dans l'axe du vaisseau. On montre alors que

$$
\bar{v}=\frac{2}{3} v_{0}
$$

tandis que le flux sanguin défini par :

$$
\Phi=\int_{0}^{d / 2} 2 \pi v(r) r d r
$$

est égal à :

$$
\Phi=\frac{\pi v_{0} d^{2}}{8}
$$

donc :

$$
\Phi=\frac{3 \pi \bar{v} d^{2}}{16} \quad \bar{v}=\frac{16 \Phi}{3 \pi d^{2}}
$$

La différence de potentiel de part et d'autre du diamètre du vaisseau est donc :

$$
\Delta V=B \bar{V} d=B \quad \frac{16 \Phi}{3 \pi d}
$$

pour un flux de $5 \mathrm{l} / \mathrm{min}=8,33.10^{-5} \mathrm{~m}^{3} / \mathrm{s}$

$$
\begin{gathered}
d=3 \mathrm{~cm}=3.10^{-2} \mathrm{~m} \\
B=1 \mathrm{~T}
\end{gathered}
$$

on obtient :

$$
\Delta \mathrm{V}=4,71 \mathrm{mV}
$$

